\title{
Prueba Electrónica Anteparto de Vitalidad Fetal
}

Dres.: Jorge A. Restrepo Salazar, Eva María Awad Zainete, Luis Fernando Uribe Vargas y Alfonso Júbiz Hasbun

\author{
UNIVERSIDAD DE ANTIOQUIA - FACULTAD DE MEDICINA \\ DEPARTAMENTO DE OBSTETRICIA Y GINECOLOGIA \\ FUNDACION HOSPITALARIA - SAN VICENTE DE PAUL
}

\section{INTRODUCCION}

El análisis de la mortalidad perinatal, la determinación del índice de Apgar, el estudio del equilibrio ácido-base del corción umbilical y las variaciones de la frecuencia cardíaca fetal, nos permiten evaluar los cuidados obstétricos inmediatos.

Se acepta que cerca del $50 \%$ de las muertes perinatales, se pueden prevenir con un buen control prenatal y con un manejo adecuado de la etapa intraparto.

Existe una relación directa entre los factores de riesgo durante la etapa preconcepcional y prenatal, con el resultado perinatal. Por lo anterior, es prioritaria la vigilancia del feto durante el embarazo y el parto, cuando la madre presenta un riesgo elevado.

En relación con la vigilancia fetal durante el embarazo, se han ensayado diferentes sistemas desde el más sencillo y simple, como la auscultación de los ruidos fetales, hasta la más sofisticada, como la fetoscopia. Sin embargo, con los avances de la electrónica, los métodos utilizados en la actualidad son simples, seguros, y a la vez, se pueden interpretar rápidamente.

En la actualidad los métodos no invasivos, fáciles, sensibles y medibles, se están imponiendo en la atención perinatal.

Si se combina el análisis de dos fenómenos importantes que se suceden durante la vida intrauterina como son la frecuencia cardíaca y los movimientos del feto, estaremos en capacidad de determinar el grado de bienestar fetal. El primero se describió desde el siglo pasado y el segundo se menciona en la Biblia, cuando se hace alusión al embarazo gemelar de Rebeca; es Ahlfel, en 1905 citado por Timor y col. (27), quien utiliza un sistema de registro para comprobar los movimientos del feto.

Diferentes autores $(2,3,18,25,26)$ han empleado la prueba bioeléctrica de vitalidad fetal durante la consulta prenatal y la recomiendan como la primera de las evaluaciones que deben efectuarse en las pacientes con factores de riesgo perinatal. 
En el presente estudio, hacemos un análisis de la prueba que denominamos, sin estímulo, que se practica en las pacientes que acuden a control prenatal.

\section{OBJETIVOS}

\section{General}

Clasificar los registros anteparto sin estímulo, efectuados a las gestantes que consultaron al Departamento de Obstetricia y Ginecología de la Facultad de Medicina de la Universidad de Antioquia, entre el 1o. de enero de 1979 y el 31 de diciembre de 1980 .

\section{Específicos}

1. Comparar los resultados del último registro anteparto, con el estado del recién nacido al minuto $y$ a los cinco minutos, de acuerdo con el índice de Apgar.

2. Relacionar el resultado del último régistro con el estado de los neonatos a las 48 horas.

3. Clasificar las distintas patologías encontradas en las gestantes estudiadas.

\section{Material y Niétodos}

Se analizaron 1.053 registros efectuados antes del parto en 435 gestantes con factores de riesgo perinatal, que consultaron al Departamento de Obstetricia y Ginecología, entre el 1o. de enero de 1979 y el 31 de diciembre de 1980.

En el presente estudio únicamente se tuvo en cuenta el último registro practicado en la consulta prenatal.

\section{Definición}

La prueba sin estímulo es un método bioeléctrico, que sirve para determinar el bienestar fetal, observando la respuesta de la frecuencia cardíaca a los movimientos espontáneos y activos.

\section{Técnica}

1. Explicación del procedimiento a la paciente.

2. En un cuarto independiente $y$ tranquilo, se coloca la gestante en posición de semi-Fowler.

3. Control inicial de la tensión arterial en decúbito lateral y sentada. Posteriormente cada 10 minutos.

4. Colocación del transductor de ultra sonido para determinar la frecuencia cardíaca fetal de base.

5. Un poco por debajo del fondo uterino, se coloca el toco-transductor.

Con el objeto de clasificar adecuadamente cada registro, nos basamos en los siguientes criterios:

1. La frecuencia cardíaca fetal de base. (F.C.F.B.) con un valor entre 120160.

2. Variabilidad latido a latido, o sea las oscilaciones rítmicas. Aceptamos como normal valores de 5 a 15.

3. Número de los movimientos fetales. (M.F.) en un período de 20 minutos; lo consideramos normal cuando es de 5 o más.

4. Ascensos. En el presente estudio aceptamos como normal variaciones entre 15 y 25 latidos. Lo consideramos como una buena respuesta del feto $y$ generalmente se producen 10 segundos después de los movimientos espontáneos. 
Figura No. 1

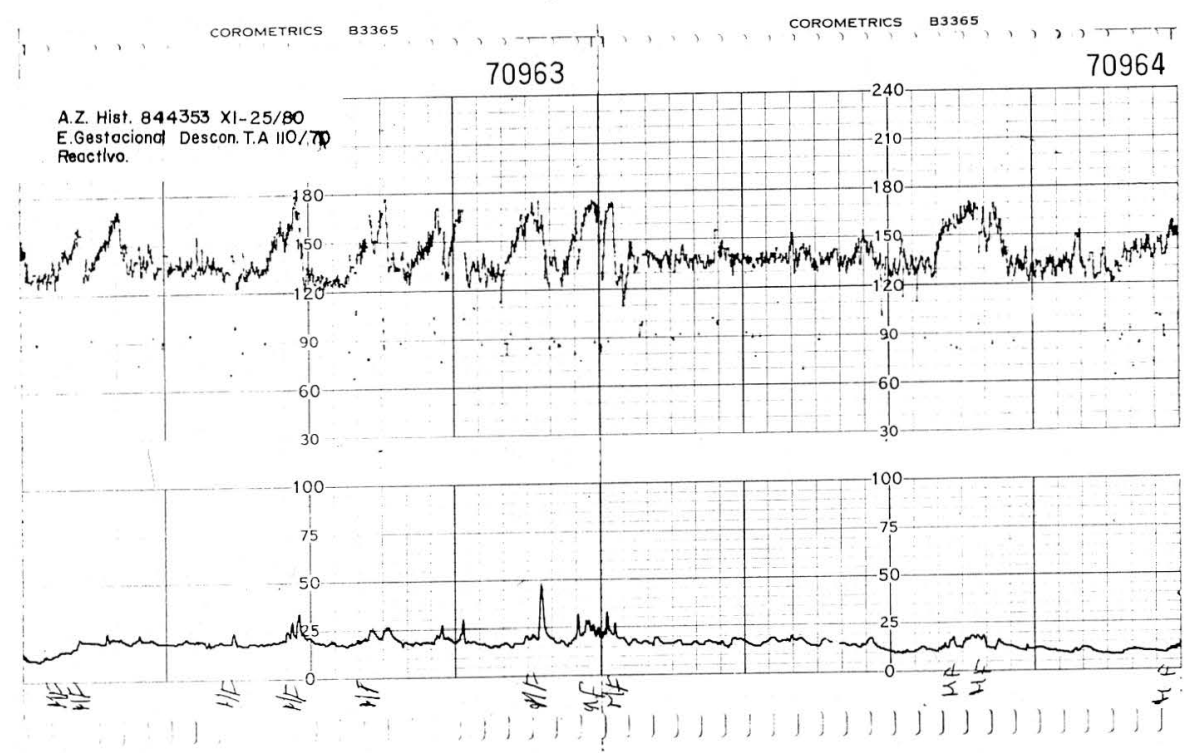

Basados en los criterios anteriores, clasificamos los registros de la siguiente manera:

A. REACTIVO: (Fig. No. 1)

1. FCFB entre 120 y 160 lat $/ \mathrm{min}$.

2. Variabilidad de 5 a $15 \mathrm{lat} / \mathrm{min}$.

3. Movimientos fetales de 5 o más en 20 minutos.

4. Ascensos de 15 lat/min. con los movimientos fetales.

B. NO REACTIVO: (Fig. No. 2)

1. FCFB entre 120 y 160 lat $/ \mathrm{min}$.

2. Variabilidad menor de $5 \mathrm{lat} / \mathrm{min}$.
Figura No. 2

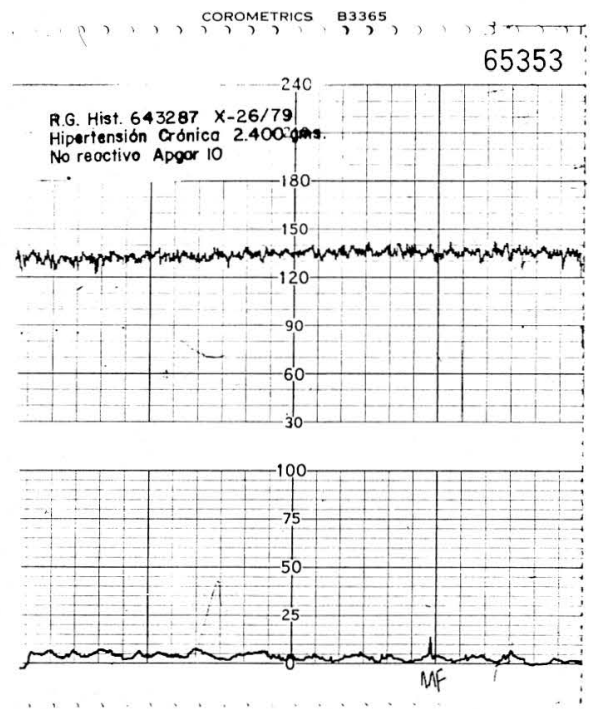


3. Menos de 5 movimientos fetales en 20 minutos.

4. Ausencia de ascensos.

\section{Figura No. 3}

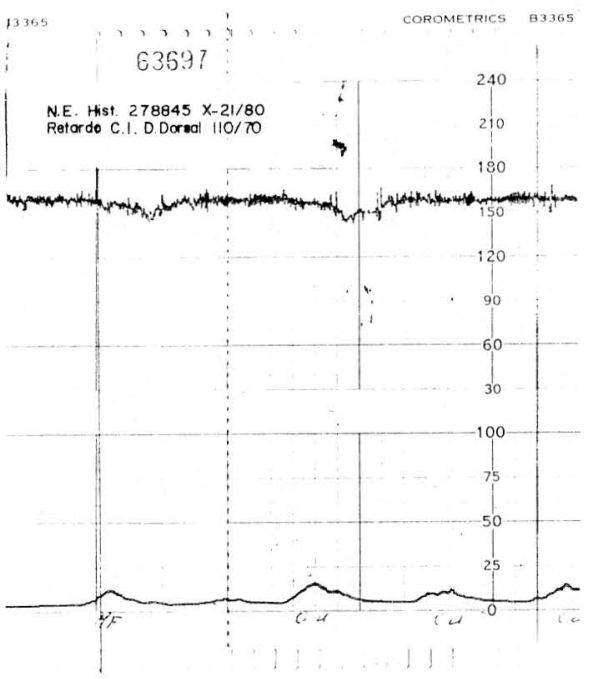

C. NO REACTIVO CON SIGNOS DE HIPOXIA: (Fig. Nos. 3, 4, 5).

En este los criterios son similares al anterior, pero se observan desaceleraciones en presencia de contracciones y movimientos espontáneos.

D. SINUSOIDAL: (Fig. No. 6).

Cuando el período de oscilaciones está superpuesto a un patrón no reactivo y la variabilidad se encuentra entre 2 y 5 lat $/ \mathrm{min}$

\section{E. NO SATISFACTORIO:}

Son aquellos registros que no permiten sacar conclusiones sobre el estado fetal $y$ que no pueden ser clasificados en ninguno de los ya mencionados.

Después de la clasificación de los registros, analizamos el estado del recién nacido, comparando el resultado del

Figura No. 4

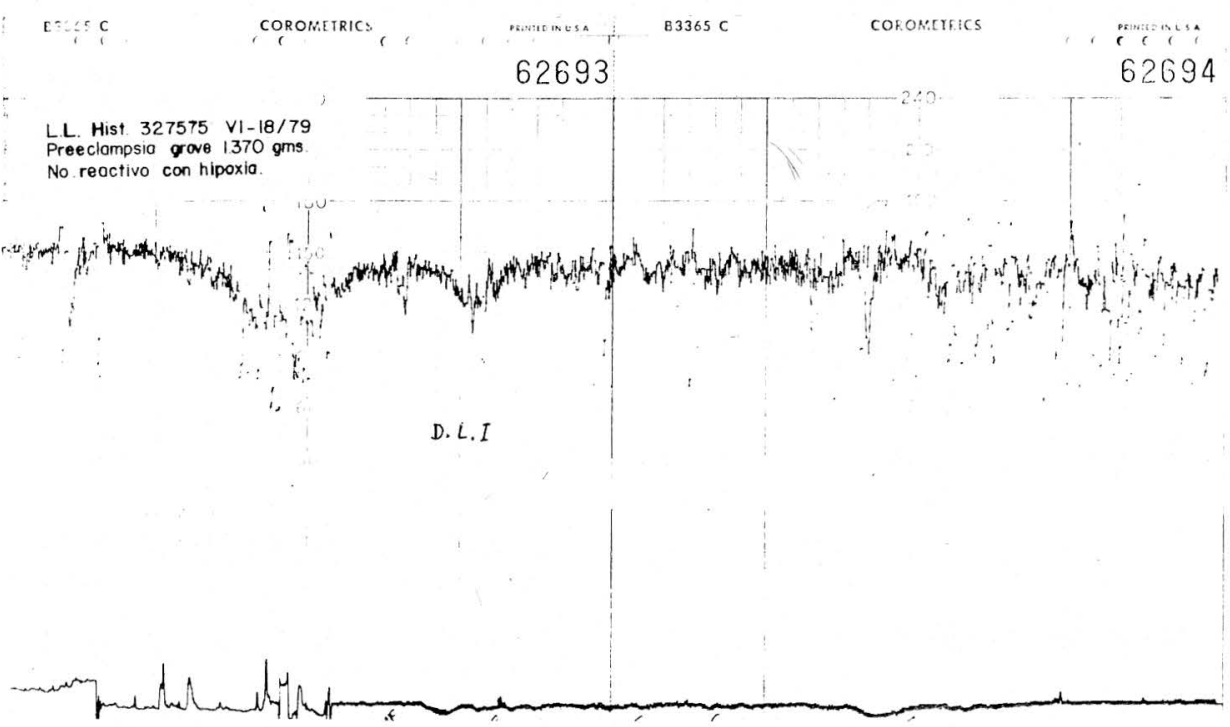




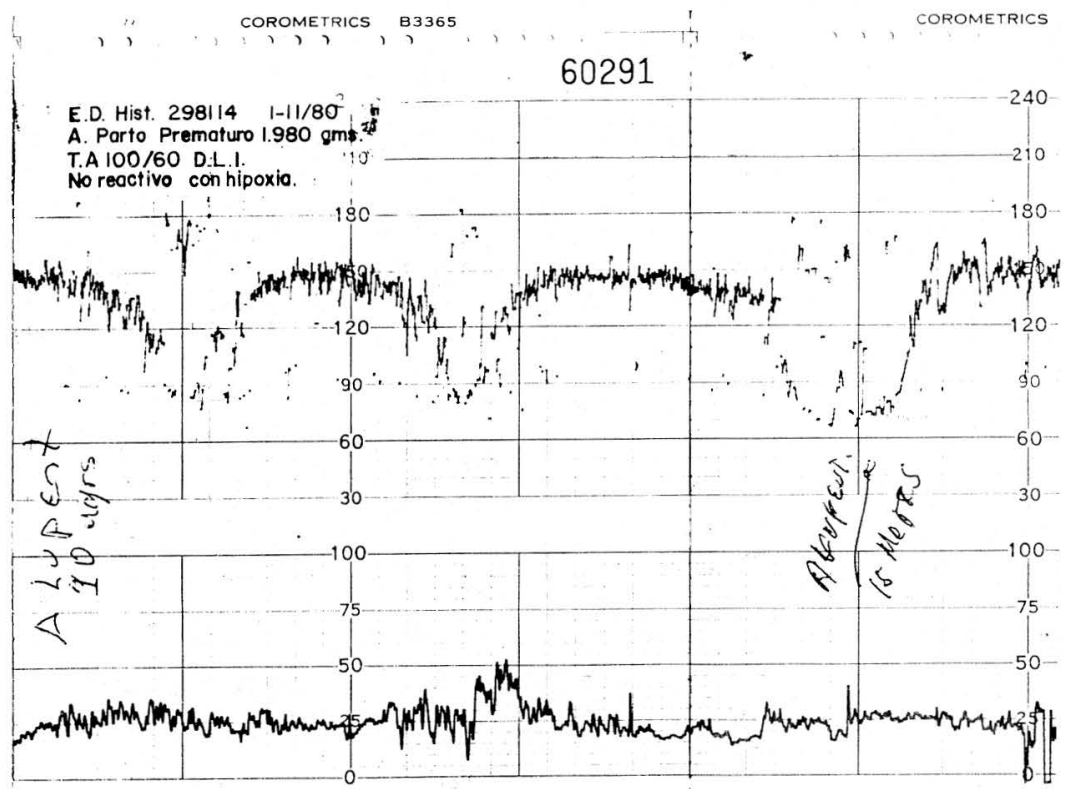

Figura No. 5

Figura No. 6

C33SS C COROMETRICS

wivtoust B3365 C COROMEIRICS

62833

62834

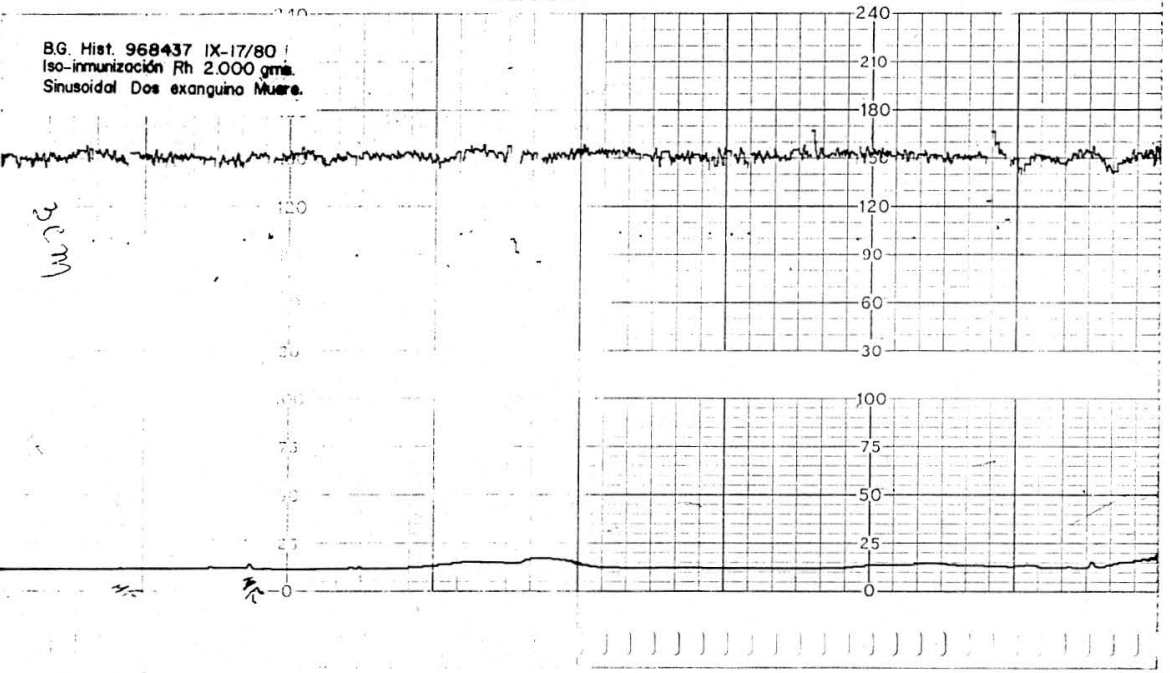


trazado con el índice de Apgar al 10. y al 5o. minuto; y también, con el estado neonatal a las 48 horas, tiempo regular que permanecen en el servicio.

Por último, se analizan los factores de riesgo de las pacientes estudiadas.

Para practicar los registros, se utilizó un monitor fetal, modelo 111, marca Corometrics.

\section{RESULTADOS}

Cuadro No. 1

\section{CLASIFICACION DE LOS REGISTROS}

\begin{tabular}{|lrr|}
\hline \multicolumn{1}{|c}{ Tipo } & No. & \% \\
\hline Reactivo & 367 & 84.4 \\
No reactivo & 16 & 3.6 \\
No reactivo con Hipoxia & 31 & 7.2 \\
Sinusoidal & 5 & 1.2 \\
No satisfactorio & 16 & 3.6 \\
\hline Total & 435 & 100.0 \\
\hline
\end{tabular}

En el cuadro anterior se puede observar el porcentaje elevado de registros reactivos.

Cuadro No. 2

RELACION ENTRE LOS REGISTROS

REACTIVOS

Y EL INDICE DE APGAR

\begin{tabular}{|lrrrr|}
\hline \multirow{2}{*}{ Apgar } & \multicolumn{2}{c}{ 1er. minuto } & \multicolumn{2}{c|}{ 5o. minuto } \\
\cline { 2 - 6 } & No. & \multicolumn{2}{c}{$\%$} & \multicolumn{2}{c|}{ No. } & $\%$ \\
\hline & & & 6 & 2.0 \\
$0-3$ & 19 & 6.2 & 3 & 1.0 \\
$4-6$ & 27 & 8.8 & 293 & 97.0 \\
$7-10$ & 259 & 85.0 & 290 \\
\hline Total & 305 & 100. & 302 & 100.0 \\
\hline
\end{tabular}

Salta a la vista la correlación que se encuentra entre el estado del recién nacido, cuando el registro es reactivo. El $85 \%$ de los neonatos al primer minuto y el $97 \%$ a los cinco minutos se encontraron vigorosos.

Se eliminaron 62 casos en los cuales no fue posible obtener el dato del estado del recién nacido. Lo anterior se explica por las dificultades locativas que impide atender el $100 \%$ de los embarazos de riesgo que son controlados en la consulta prenatal.

Cuadro No. 2A.

ESTADO DE LOS NEONATOS A

$$
\text { LAS } 48 \text { HORAS }
$$

SEGUN LOS REGISTROS REACTIVOS

\begin{tabular}{|lrr|}
\hline \multicolumn{1}{|c}{ Estado } & No. & \multicolumn{1}{c|}{$\%$} \\
\hline Nacidos muertos & 6 & 2.0 \\
Buenas condiciones & 291 & 95.7 \\
Muertos & 7 & 2.3 \\
\hline Total & 304 & 100.0 \\
\hline
\end{tabular}

Se observa que a las 48 horas el $95.7 \%$ de los neonatos salen del Hospital en buenas condiciones.

Cuadro No. 3

RELACION ENTRE LOS REGISTROS NO REACTIVOS Y EL INDICE DE APGAR

\begin{tabular}{|ccccc|}
\hline \multirow{2}{*}{ Apgar } & \multicolumn{2}{c}{ ler. minuto } & \multicolumn{2}{c|}{ 5o. minuto } \\
\cline { 2 - 5 } & No. & $\%$ & No. & $\%$ \\
\hline $0-3$ & 2 & 16.7 & 1 & 8.3 \\
$4-6$ & 2 & 16.7 & 0 & 0 \\
$7-10$ & 8 & 66.6 & 11 & 91.7 \\
\hline Total & 12 & 100. & 12 & 100.0 \\
\hline
\end{tabular}


Llama la atención que al primer minuto el $66 \%$ de los neonatos se encontraban vigorosos y que a los cinco, prácticamente el $92 \%$ estaban en buenas condiciones. Lo anterior nos hace pensar que no siempre un registro clasificado como no reactivo, puede ser concluyente para determinar el estado del feto.

\section{Cuadro No. 3A}

\section{ESTADO DE LOS NEONATOS A LAS 48 HORAS SEGUN LOS REGISTROS NO REACTIVOS}

\begin{tabular}{|lrr|}
\hline Estado & No. & $\%$ \\
\hline Buenas condiciones & 11 & 91.7 \\
Muertos & 1 & 8.3 \\
\hline Total & 12 & 100.0 \\
\hline
\end{tabular}

Como se comentó en el cuadro anterior, el registro no reactivo no predijo el resultado de los neonatos, toda vez que más de las dos terceras partes de éstos salieron en buenas condiciones.

\section{Cuadro No. 4}

\section{RELACION ENTRE LOS REGISTROS NO REACTIVOS CON HIPOXIA $Y$ EL INDICE DE APGAR}

\begin{tabular}{|lrrrr|}
\hline Apgar & \multicolumn{2}{c|}{ 1er. minuto } & \multicolumn{2}{c|}{ 5o. minuto } \\
\hline & No. & $\%$ & No. & \multicolumn{1}{c|}{$\%$} \\
\cline { 2 - 5 } $0-3$ & 8 & 30.8 & 2 & 7.7 \\
$4-6$ & 6 & 23.0 & 6 & 23.0 \\
$7-10$ & 12 & 46.2 & 18 & 69.3 \\
\hline Total & 26 & 100.0 & 26 & 100.0 \\
\hline
\end{tabular}

Aproximadamente el $50 \%$ de los recién nacidos al primer minuto se encontraban en buenas condiciones y a los cinco minutos el $70 \%$ estaban vigorosos.
Los resultados son semejantes a los encontrados en los registros no reactivos.

Cuadro No. 4A.

\section{ESTADO DE LOS NEONATOS A LAS 48 HORAS SEGUN LOS REGISTROS \\ NO REACTIVOS CON HIPOXIA}

\begin{tabular}{|lrc|}
\hline Estado & No. & $\%$ \\
\hline Buenas condiciones & 18 & 72 \\
Muertos & 7 & 28 \\
\hline Total & 25 & 100 \\
\hline
\end{tabular}

El número de neonatos que salen en buenas condiciones del Hospital es igual a aquellos recién nacidos que se encontraban vigorosos a los cinco minutos.

Cuadro No. 5

\section{RELACION ENTRE LOS REGISTROS SINUSOIDALES Y EL INDICE DE APGAR}

\begin{tabular}{|ccccc|}
\hline Apgar & \multicolumn{2}{l}{ 1er. minuto } & \multicolumn{2}{c|}{ 5o. minuto } \\
\hline & No. & $\%$ & No. & $\%$ \\
\cline { 2 - 5 } $0-3$ & 4 & 80.0 & 2 & 40.0 \\
$4-6$ & 1 & 20.0 & 2 & 40.0 \\
$7-10$ & 0 & 0 & 1 & 20.0 \\
\hline Total & 5 & 100.0 & 5 & 100.0 \\
\hline
\end{tabular}

Se encontró que el resultado del registro se relaciona con el estado de los recién nacidos al minuto y a los cinco minutos en todos los casos. Es de anotar que las gestantes estaban sensibilizadas al factor Rh, y los neonatos presentaron enfermedad hemolítica. A las 48 horas, todos los neonatos habían muerto. 
Cuadro No. 6

\section{RELACION ENTRE LOS REGISTROS NO SATISFACTORIOS $Y$ EL INDICE DE APGAR}

\begin{tabular}{|c|c|c|c|c|}
\hline \multirow[t]{2}{*}{ Apgar } & \multicolumn{2}{|c|}{ 1er. minuto } & \multicolumn{2}{|c|}{ 5o. minuto } \\
\hline & No. & $\%$ & No. & $\%$ \\
\hline $0-3$ & 2 & 13.3 & 1 & 6.7 \\
\hline $4-6$ & 2 & 13.3 & 0 & 0 \\
\hline $7-10$ & 11 & 73.4 & 14 & 93.3 \\
\hline Total & 15 & 100.0 & 15 & 100.0 \\
\hline
\end{tabular}

Cuadro No. 6A.

\section{ESTADO DE LOS NEONATOS A LAS 48 HORAS SEGUN LOS REGISTROS NO SATISFATORIOS}

\begin{tabular}{|lrr|}
\hline Estado & No. & $\%$ \\
\hline Buenas condiciones & 14 & 93.3 \\
Muertos & 1 & 6.7 \\
\hline Total & 15 & 100.0 \\
\hline
\end{tabular}

Los registros no satisfactorios impiden hacer un pronóstico del estado del recién nacido o del neonato. Pueden existir otros factores diferentes al estado fetal, que dificultan la realización del registro o su adecuada interpretación.

De las 435 pacientes en control de Alto Riesgo Obstétrico, se encontraron con una sola patología 409 y con 2 o más 26.

\section{Discusión}

La vigilancia bioeléctrica del feto es un método incuestionable durante la
Tabla No. 1

\section{PATOLOGIAS ASOCIADAS}

\begin{tabular}{|lll|}
\hline 1. Enfermedad hipertensiva del & \\
2. Embarazo & 78 \\
3. Hipertensión crónica & 48 \\
4. Edad gestacional desconocida & 40 \\
5. Rh sensibilizado & 26 \\
6. Diabetes & 19 \\
7. Cesáreas anteriores & 16 \\
8. Amenaza de parto prematuro & 14 \\
9. Antecedentes de prematuros & 13 \\
10. Cardiopatías & 12 \\
\hline
\end{tabular}

atención del proceso reproductivo $(4,5$, $6,7,9,11,25,26)$. En el presente estudio se observa que cuando el último registro sin estímulo realizado antes del parto es reactivo, el $97 \%$ de los recién nacidos se encuentran vigorosos. Lo anterior ha sido suficientemente comprobado por varios investigadores, 14,10 , $13,14,16,21)$. A las 48 horas, el $95 \%$ de los neonatos abandonaron el Hospital en buenas condiciones.

Cuando la prueba sin estímulo se clasifica como no reactiva, diferentes autores recomiendan continuar con la estimulación de oxitocina $(6,8,15,21)$. Sin embargo, nosotros eliminamos los registros con oxitocina y sólo relacio. namos la prueba sin estímulo con el estado del recién nacido al primer minuto, a las 5 y a las 48 horas de vida. De los 12 registros clasificados como no reactivo, el $61 \%$ de los recién nacidos al primer minuto y el $91.7 \%$ a los cinco, presentaron un índice de Apgar de 7 o más, $y$ a las 48 horas sólo había fallecido un neonato. Estamos de acuerdo con los autores que recomiendan realizar una prueba de oxitocina, siempre que el re- 
gistro sin estímulo no presente aceleraciones con los movimientos fetales. (6, $8,11,12,21)$.

La presencia de desaceleraciones variables con los movimientos espontáneos del feto, es un signo de disminución de la reserva fetal. Sólo el $46 \%$ de los recién nacidos al primer minuto y el $69 \%$ a los cinco, presentaron un índice de Apgar de 7 o más. El $48 \%$ de los neonatos tallecieron en las primeras 48 horas de vida. O'Leary y colaboradores (17). consideran que existe una desaceleración variable cuando la amplitud es mayor de $10 \mathrm{lat} / \mathrm{min}$., la duración es superior a 10 segundos y el número de estos cambios es 3 o más. La presencia de desaceleraciones variables en un registro sin estímulo, afecta en forma adversa el resultado perinatal.

En 5 embarazadas sensibilizadas al factor $R h$, el registro anteparto fue clasificado como sinusoidal. A los cinco minutos sólo uno de los recién nacidos alcanzó un índice de Apgar de 7 o más. A las 48 horas todos los neonatos habian fallecido. Estos datos son similares a los encontrados por Rochard (22) cuando analiza 20 registros sinusoidales de madres $R$ h negativas sensibilizadas.

Cuando los registros se clasifican como insatisfactorios, no es posible definir una conducta en relación con el estado fetal. La mayoría de las veces se debe a obesidad de la madre o a dificultades técnicas. Siempre se recomienda repetir la prueba en las siguiente 48 horas. El $93 \%$ de los recién nacidos de este grupo se encontraron vigorosos a los cinco minutos y abandonaron el Hospital en buenas condiciones.

Existen diferentes clasificaciones de la prueba sin estímulo para la vigilancia fetal anteparto $(1,18,19,25,29)$. La que se presenta en este trabajo combina algunas de ellas por su facilidad de interpretar y su relación con el estado de los neonatos.
Los métodos bioeléctricos de vigilancia fetal durante el embarazo y el parto, están indicados en todos los embarazos con factores de riesgo, especialmente de origen placentario.

La actividad espontánea del feto registrada clínica o electrónicamente (20, $23,24,27)$, debe recomendarse en forma rutinaria en todos los embarazos durante el control prenatal. Trudinger y Boyland (28), recomiendan la estimulación con el sonido para registrar los cambios de la frecuencia cardíaca fetal.

Otro fenómeno que es fácil de registrar y de gran valor pronóstico sobre el estado del feto, es el relacionado con las aceleraciones (29).

\section{Resumen y Conclusiones}

Se analizaron 1.053 registros anteparto de pacientes con factores de riesgo perinatal y se seleccionaron 435 correspondientes al último control realizado durante la consulta prenatal. El $84.4 \%$ de los registros fueron clasificados como reactivos, $7.2 \%$ no reactivos con hipoxia, $3.6 \%$ no reactivos y $1.2 \%$ sinusoidal.

En el grupo de registros reactivos el $97 \%$ de los recién nacidos presentaron un Apgar de 7 o más a los cinco minutos. En los no reactivos el $92 \%$ estaban vigorosos a los cinco minutos. Cuando la desaceleración estuvo presente en el registro nc reactivo, sólo el $69.6 \%$ de los neonatos se encontraron vigorosos a los cinco minutos. En los 5 trazados sinusoidales sólo un recién nacido se encontró vigoroso a los cinco minutos $y$ todos murieron en las primeras 48 horas.

1. La prueba sin estímulo reactiva se correlacionó con el estado del neonato, al primero $y$ al quinto minuto, $y$ a las 48 horas. 
2. Cuando el registro es clasificado como no reactivo, debe continuarse la estimulación con oxitocina.

3. En los registros no reactivos, cuando se sobrepone la desaceleración variable el resultado perinatal es pobre $(69 \%)$.
4. El trazado de tipo sinusoidal predice un resultado perinatal malo y se encuentra especialmente en la isoinmunización al factor $\mathrm{Rh}$.

\section{ANTEPARTUM ELECTRONIC TEST FOR FETAL VITALITY}

\section{Summary and conclusions}

This study analyzed 1.053 antepartum medical records of patients with perinatal risk factors. Of these, 435 were selected in their last check-up during antenatal care; $84.4 \%$ of the records were classified as reactive, $7.2 \%$ as nonreactive with hypoxia, $3.6 \%$ non-reactive and $1.2 \%$ sinusoid.

Of the reactive records, $97 \%$ of the newborns had an Apgar of 7 or more five minutes after.

Among the non-reactive, $92 \%$ were vigorous after five miriutes. Whenever there was deceleration in the non-reactive group, only $69.6 \%$ were vigorous after five minutes. Among the 5 sinusoid

\section{Bibliografía}

1. AMANKWAH, K.S. et al. A New Definition of the Nonstress Test. Obstet. Gynecol. 55 (5): 49-51, Jul. 1980.

2. BARRADA, M.I. EDWARDS. L.E. and HAKANSON. E.Y. Antepartum Fetal Testing. I The Oxytocin Challenge Test. Am. J. Obstet. Gynecol. 134 (5): 532-37, 1979

3. Idem et al. Antepartum Fetal Testing. II The Aceleration Constant Ratio: A traces, only one newborn was vigorous after five minutes, and all died within the first 48 hours.

1. The reactive test without stimulus was correlated with the general condition of the newborn during the first and fifth minute and after 48 hours.

2. When the record is classed as nonreactive, the stimulation should continue by means of oxitoxin.

3 . For the non-reactive records, whenever variable deceleration is present, perinatal results are poor $(69 \%)$.

4. A sinusoid trace anticipates a bad perinatal result and is most frequently present in $\mathrm{Rh}$ isoimmunization.

Nonstress Test. Am. J. Obstet. Gynecol. 134 (5): 538-543, 1979.

4. FLYNN. A.M. and KELLY. J. Evaluation of Fetal Wellbeing by Antepartum Fetal Heart Monitoring. Br. Med. J. 1: 93639, 1977.

5. GARITE, T.J. and FREEMAN, R.K. Prueba para la vigilancia del sufrimiento fetal antes del parto. Ginecología y Obstetricia. Temas Actuales. 2: 297--309, 1979. 
6. GIBBONS, J.M. Jr. NAGLE. P. Correlation of Nonstressed Fetal Hearth Rate with Sequential Contraction Stress Test. Obstet. Gynecol. 55 (5): 612-16, 1981.

7. HILL, M.L.M. Diagnosis and Management of Fetal Distress. Mayo Clin. Proc. 54: 784-93, 1979.

8. HUDDLESTON, J.F. et al. Oxytocin Challenge Test for Antepartum Fetal Assessment. Report of a Clinical Experience. Am. J. Obstet. Gynecol. 135 (5): 609-14, 1979.

9. INGARDIA, Ch. J. et al. Prognostic Components of the Non reactive Nonstress Test. Obstet. Gynecol. 56 (3): 305-10, 1980.

10. LEE, C.Y. and DRUKKEN, B. The Nonstress for the Antepartum Assessment of Fetal Reserve. Am. J. Obstet. Gynecol. 134 (4): 460-70, 1979.

11. Iden et al. Fetal Activity Acceleration Determination for the Evaluation of fetal Reserve. Obstet. Gynecol. 48 (1): 1926, 1976.

12. LEON, J. Fetal Movements Associated with Fetal Heart Rate Accelerations and Decelerations. Am. J. Obstet. Gynecol. 134 (6): 724-25, 1979.

13. MENDENHALL, H.W. et al. The Nonstress Test: The Value of a Simple Acceleration in Evaluating the Fetus at Risk. Am. J. Obstet. Gynecol. 136 (1): 87-91, 1980.

14. NOCHIMSON, D.J. et al. The Nonstress Test. Obstet. Gynecol. 51 (4): 419-21, 1978.

15. NORIEGA, L. et al. Pruebas de Tolerancia Fetal. Ginecol. Obstet. Mex. 38 (230): 435-42, 1975.

16. ODENDAAL, $H$. and SANDENBERCH. H.A. Acceleration Patterns of the Fetal
Heart Rate Beofore and During Labour. Obstet. Gynecol. Surv. 33 (5): 323-24, 1978.

17. O'LEARY, J.A. ANDRINOPOULOS, G. C. and GIORDANO, P.C. Variable Decelerations and the Nonstress Test: An Indication of Cord Compromise. Am. J. Obstet. Gynecol. 137, 704, 1980.

18. PAUL, R.H. and KEEGAN, K.A. Vigilancia fetal antes del parto mediante pruebas sin esfuerzos. Ginecol. Obstet. Temas actuales. 2, 353-60, 1979.

19. Idem, and MILLER, F.C. Vigilancia dela frecuencia del corazón fetal antes del parto. En riesgo al to en Obstetricia. Clin. Obstet. Ginecol. 2: 401-11, 1978.

20. PEARSON, J.F. and WEAVER, J.B. Fetal Activity and Fetal Wellbeing: An Evaluation. Br. Med. J. 1: 1.305-07, 1976.

21. PRATT. D. et al. Fetal Stress and Nonstress Test: An Analysis and Comparasion of their Ability to Identily Fetal Outcome. Obstet. Gynecol. 54 (4): 419-23, 1979.

22. ROCHARD, F. et al. Nonstressed Fetal Heart Rate Monitoring in the Antepartum Period. Am. J. Obstet. Gynecol. 126 (6): 699-706, 1976.

23. ROSEN. M.G. et al. Vigilancia del Movimiento Fetal. Ginecol. Obstet. Temas Actuales. 2: 327-36, 1979.

24. SADOUSKY. E. and YAFFE, H. DAILY Fetal Movement Recording and Fetal Prognosis. Obstet. Gynecol. 41: 845-50, 1973.

25. SCHIFRIN, B.S. et al. Routine Fetal Heart Rate Monitoring in the Antepartum Period. Obstet. Ginecol. 54 (1): 21-25 1979. 
26. Idem Antepartum Fetal Heart Rate Monitoring. In Intrauterine Asphyxia and the Developing Fetal Brain. Edited By Gluck, L. Chicago. Year Book Medical Pub. 1977. p. 205-24

27. TIMON-TRITSCH. I.E. et al. Movimientos fetales: Un breve repaso. Clin. Obstet. Gynecol. 3: 601-602-610, 1979.
28. TRUDINGER. B.J. and BOYLAND, P. Antepartum Fetal Heart Rate Monitoring: Value of Sound Stimulation. Obstet Gynecol. 55 (4): 265-68, 1980.

29. WOOD, C. et al. Acceleration of the Fetal Heart Rate. Am. J. Obstet. Gynecol. 134 (5): 523-27. 1979. 